\title{
BMJ Open Are ethnic differences, urinary iodine status, lead and cadmium exposure associated with thyroid autoimmunity and hypothyroid status? A cross- sectional study
}

\author{
Yi Chen, ${ }^{1}$ Qian Xiang, ${ }^{2}$ Ningjian Wang, ${ }^{1}$ Wen Zhang, ${ }^{1}$ Chunfang Zhu, ${ }^{1}$ \\ Yuying Wang, ${ }^{1}$ Heng Wan, ${ }^{1}$ Jing Cheng, ${ }^{1}$ Kun Zhang, ${ }^{1}$ Yan Cai, ${ }^{2}$ Yingli Lu (D) ${ }^{1}$
}

To cite: Chen $\mathrm{Y}$, Xiang $\mathrm{Q}$, Wang N, et al. Are ethnic differences, urinary iodine status, lead and cadmium exposure associated with thyroid autoimmunity and hypothyroid status? A crosssectional study. BMJ Open 2022;12:e056909. doi:10.1136/ bmjopen-2021-056909

- Prepublication history for this paper is available online. To view these files, please visit the journal online (http://dx.doi. org/10.1136/bmjopen-2021056909).

$\mathrm{YC}$ and $\mathrm{QX}$ contributed equally.

Received 02 September 2021 Accepted 28 January 2022

\section{Check for updates}

(c) Author(s) (or their employer(s)) 2022. Re-use permitted under CC BY-NC. No commercial re-use. See rights and permissions. Published by BMJ.

${ }^{1}$ Institute and Department of Endocrinology and Metabolism, Shanghai Ninth People's Hospital, Shanghai JiaoTong University School of Medicine, Shanghai, China

${ }^{2}$ Department of Endocrinology, the Fifth affiliated Hospital of Kunming Medical University, Yunnan Honghe Prefecture Central Hospital (Ge Jiu People's Hospital), Yunnan, China

Correspondence to

Professor Yingli Lu;

luyingli2008@126.com and

Yan Cai; caiyan160@126.com

\section{ABSTRACT}

Objective We aimed to evaluate the effects of different ethnicities and potential environmental exposure on the prevalence of thyroid autoimmune status and hypothyroid status.

Design The data were obtained from two cross-sectional studies.

Participants 2105 participants in Shanghai (Han) and 772 participants in Yunnan Honghe Prefecture (Han, Yi, Miao and Hani), aged 18-75 were enrolled.

Methods Participants underwent several checkups, including urinary iodine concentration, blood lead (BPb) and blood cadmium (BCd), thyroid peroxidase antibody (TPOAb), thyroglobulin antibody ( $\mathrm{TgAb}$ ), thyroid stimulating hormone (TSH) as well as thyroid ultrasonography (US). Thyroid autoimmune status was defined as: antithyroid antibody positive (ATA+): TPOAb + or TgAb+; and ATA + and US+: TPOAb + or TgAb + together with characteristic US features. Results The standardised prevalence of thyroid autoimmune positivity in Yunnan were higher than those in Shanghai (TPOAb+: $13.56 \%$ vs $8.27 \%, p<0.001$; TgAb+: $9.28 \%$ vs $7.09 \%, p=0.045 ;$ ATA $+: 16.96 \%$ vs $11.10 \%, p<0.001 ;$ ATA + and US+: $8.96 \%$ vs $6.64 \%$, $\mathrm{p}=0.036)$. For urinary iodine-to-creatinine ratio $(\mathrm{UI} / \mathrm{Cr})$, compared with the level of $100.00-199.99 \mu \mathrm{g} / \mathrm{g}$, the level of $\geq 300.00 \mu \mathrm{g} / \mathrm{g}$ had a 1.5 -fold risk for ATA + and US+ (OR 1.455, $p=0.041$ ). The levels of 200.00-299.99 $\mu \mathrm{g} / \mathrm{g}$ and $\geq 300.00 \mu \mathrm{g} / \mathrm{g}$ were positively associated with hypothyroid status (OR 1.509, $p=0.002$ and OR 1.338 , $p=0.043)$. Compared with the first quartiles, the fourth quartiles of $\mathrm{BPb}$ were positively associated with $\mathrm{TPOAb}+$ (OR 1.637, p=0.006), ATA+ (OR 1.435, p=0.025), ATA + and US+ (OR 1.641, $p=0.013)$, hypothyroid status (OR 1.467, $p=0.013$ ) and TSH levels ( $B$ 0.092, $p=0.021$ ). The fourth quartile of $\mathrm{BCd}$ was positively associated with the prevalence of $A T A+(0 R 1.427, p=0.036)$.

Conclusions Higher levels of $\mathrm{UI} / \mathrm{Cr}, \mathrm{BPb}$ and $\mathrm{BCd}$ may be associated with thyroid autoimmunity and hypothyroid status.

\section{INTRODUCTION}

Autoimmune thyroid diseases (AITDs) are the most frequent autoimmune disorders in

\section{Strengths and limitations of this study}

- The current study was performed in a general population instead of a clinic-based population, making the results potentially more generalisable.

- The data were highly reliable because all anthropometric measurements, questionnaires and ultrasonography were completed by the same trained research group with strong quality control, which minimising the inter-individual errors.

- Because of the cross-sectional nature of the study, no causal inferences can be drawn.

- Since the sample size of four different ethnics in Yunnan was small, this pure exploratory research could be regarded as a pilot experiment.

- Blood cadmium and blood lead concentrations were based on a single blood sample, and they may represent short-term exposure.

humans and the most common pathological status in thyroid gland. ${ }^{1}$ The prevalence of AITD is estimated to be $5 \%$ in the $\mathrm{USA}^{2}$; however, the prevalence of higher antithyroid antibodies (ATAs) without clinical manifestations may be higher. ${ }^{3}$ Either thyroid peroxidase antibody (TPOAb) or thyroglobulin antibodies (TgAb), which serve as a clinical marker for detecting AITD, ${ }^{4-6}$ can be found in approximately $20 \%$ of the general population.

The presence of ATAs, mainly TPOAb, may indicate the presence of AITD, which is closely associated with thyroid dysfunction. ${ }^{8}$ Recently, a study suggested that thyroid autoimmunity was positively associated with glycated haemoglobin, homeostasis model assessment of insulin resistance (HOMA-IR), obesity, central obesity, hyperlipidaemia and metabolic syndrome, especially in women. ${ }^{9}$ The positivity of TPOAb and TgAb is also 
positively associated with the prevalence of non-alcoholic fatty liver disease. ${ }^{10}$

Thus, issues on thyroid autoimmunity have raised more attention. Genetic backgrounds, such as ethnic or race differences, are approximately $73 \%$ responsible for the presence of TPOAb and TgAb, ${ }^{11}$ while environmental factors such as iodine status, smoking and drugs contribute to the occurrence of thyroid autoimmunity positivity for approximately $20 \% .{ }^{12}{ }^{13}$ Few studies have simultaneously reported the relationship of these risk factors (ethnic or race differences and environmental exposure) with thyroid autoimmunity. In the current exploratory study, we used the data from two ongoing cross-sectional studies to evaluate (1) the different prevalence of thyroid autoimmunity and hypothyroid status between two different regions (Shanghai and Yunnan) and among four ethnic groups (Han, Yi, Miao and Hani); and (2) the potential relationship of the slight difference in the concentration of environmental exposures (including iodine, lead $(\mathrm{Pb})$ and cadmium $(\mathrm{Cd}))$ and the prevalence of thyroid autoimmunity and hypothyroid status. Understanding the epidemiology of thyroid autoimmunity, including its geographical differences and environmental influences, may provide clues to find its aetiology and a new strategy for thyroid autoimmunity-related glucose and lipid metabolism disorders.

\section{MATERIALS AND METHODS \\ Study participants}

The data of the current study were obtained from two ongoing cross-sectional studies. One is the SPECT-China 2 study, which is a population-based survey on the prevalence of metabolic diseases and risk factors in East China. The approval number of the SPECT-China 2 study is ChiCTR-ECS-1900021356 which was registered at www. chictr.org.cn (The WHO international clinical trials registered organisation registered platform). Another cross-sectional study was performed in South Yunnan (a multiethnic region) in Southwest China in 2019 and was designed to investigate the association between vitamin $\mathrm{D}$ and the prevalence of glucose and lipid metabolism diseases in South Yunnan. The ethnic information of each participant was obtained from his/her own citizen's identity card of the People's Republic of China. All participants provided written informed consent before data collection. All procedures followed were in accordance with the ethical standards of the responsible committee on human experimentation (institutional and national) and with the Helsinki Declaration of 1975, as revised in 2008.

In this study, a stratified cluster sampling method was used. In Huangpu District, Shanghai, three sites were randomly selected and in Honghe Prefecture, Yunnan Province, three ethnic minority villages (Yi, Miao and Hani people gathering areas) and one urban area (Han people gathering area) were randomly selected. Inclusion criteria were Chinese citizens aged $18-75$ years old

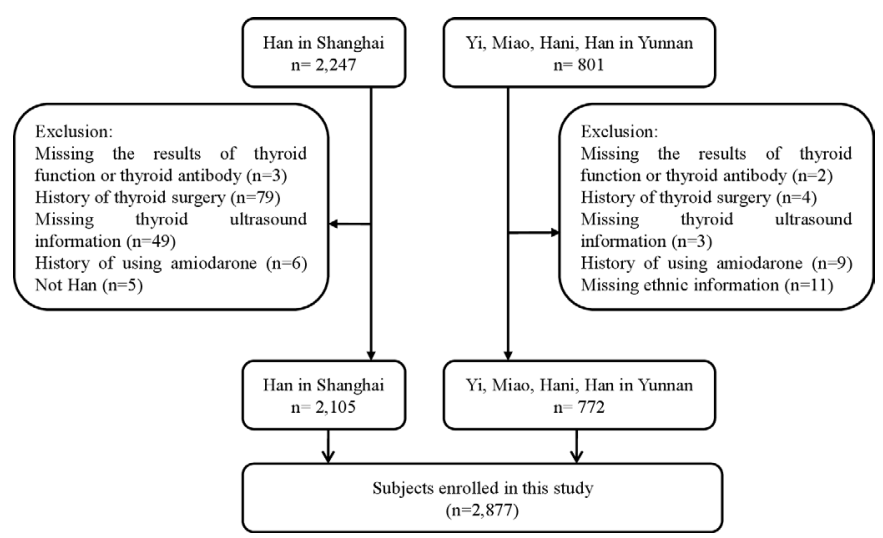

Figure 1 Flowchart of participants' inclusion and exclusion.

and had lived at their current residence for 6 months or longer. Exclusion criteria were those with severe communication problems, with acute illness or who were unwilling to participate. Initially 2247 participants in Shanghai (Han) and 801 participants in Yunnan Honghe Prefecture (Han, Yi, Miao and Hani) were enrolled. We then excluded participants who were missing the results of thyroid function (Serum thyroid stimulating hormone (TSH), total triiodothyronine $\left(\mathrm{T}_{3}\right)$ or total thyroxin $\left(\mathrm{T}_{4}\right)$ ) or thyroid antibody (TPOAb or TgAb) (n=5, 3 in Shanghai and 2 in Yunnan), had a history of thyroid surgery $(n=83$, 79 in Shanghai and 4 in Yunnan), missing thyroid ultrasound (US) information $(n=52,49$ in Shanghai and 3 in Yunnan), had a history of using amiodarone ( $\mathrm{n}=13,6$ in Shanghai and 9 in Yunnan) and missing ethnic information or not Han in Shanghai $(n=16,5$ in Shanghai and 11 in Yunnan) in this study. Finally, 2877 participants (2105 in Shanghai and 772 in Yunnan) were involved in the current study. Figure 1 shows the inclusion and exclusion of participants in this analysis. Details of the participants are described in table 1.

\section{Data collection}

All steps of the investigation processes in Shanghai and Yunnan were carried out by the same research team according to the same protocol. A standard questionnaire was administered by trained staff to obtain information on demographic characteristics, personal and family medical history and risk factors in their daily lives. All anthropometric measurements were conducted at the same time when blood samples and urinary samples were collected.

\section{Blood and urinary sample assays}

Fasting blood samples for laboratory assays were obtained by venipuncture from 07:00 to 10:00. Blood samples were stored at $2^{\circ} \mathrm{C}-8^{\circ} \mathrm{C}$ when collected and shipped to local central laboratories, which were certified by the College of American Pathologists, within 2-4 hours of collection. On the day of blood sample collection, as soon as the blood samples arrived in the laboratory, serum separation and sample testing began. Samples testing included serum TPOAb, TgAb, TSH, $\mathrm{T}_{3}$ and $\mathrm{T}_{4}$ and whole-blood $\mathrm{Pb}$ and Cd; electrochemiluminescence (Roche, E601, Germany) 


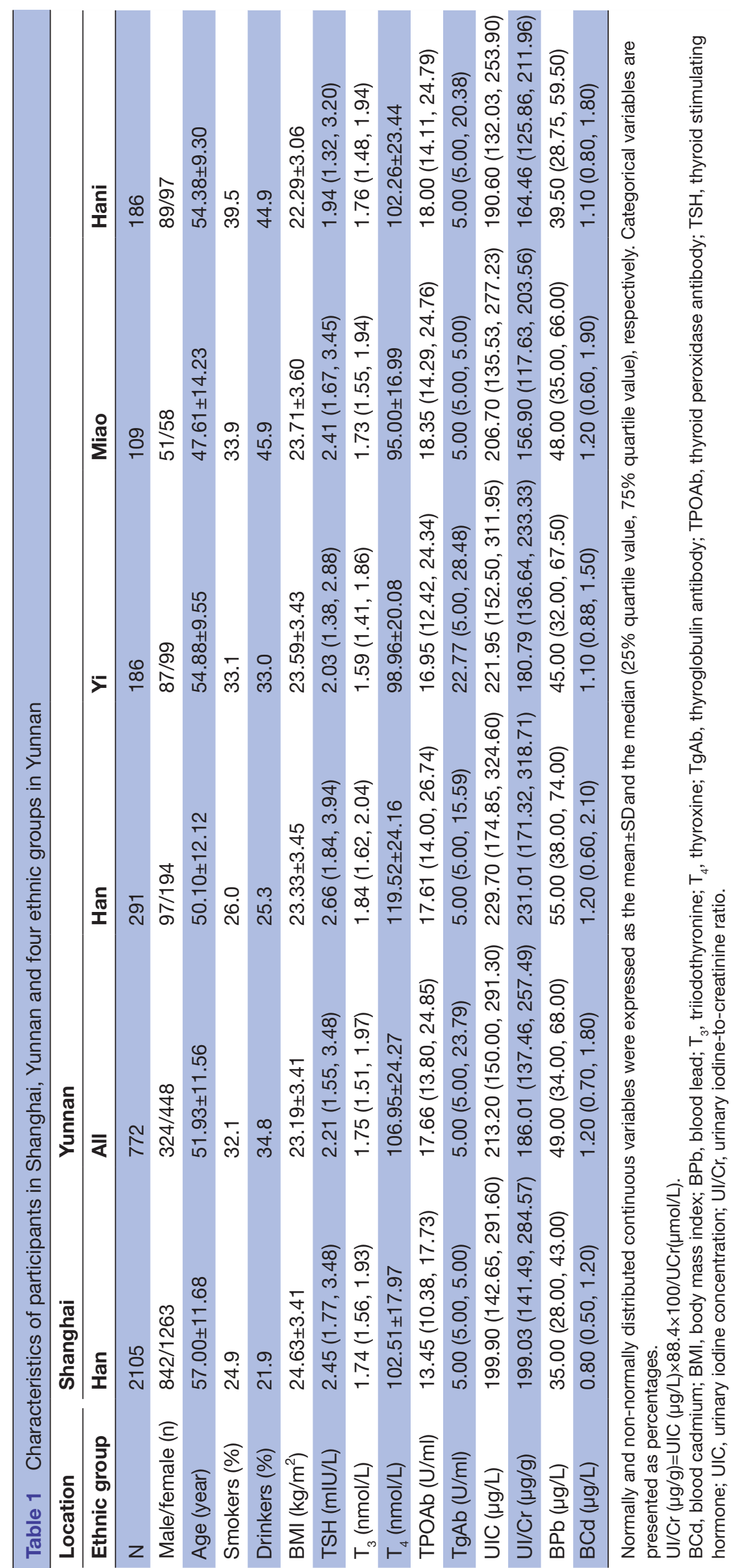


was used to measure TSH, $\mathrm{T}_{3}, \mathrm{~T}_{4}, \mathrm{TPOAb}$ and TgAb. (1) TSH: A sandwich principle was used. Samples, biotinylated monoclonal TSH-specific antibodies and monoclonal TSH-specific antibodies labelled with ruthenium complex reacted to form sandwich complex. After addition of streptavidin-coated microparticles, the complex became bound to the solid phase via interaction of biotin and streptavidin. The reaction mixture was aspirated into the measuring cell where the microparticles were magnetically captured onto the surface of the electrode. Unbound substances were then removed with ProCell/ ProCell M. Application of a voltage to the electrode then induced chemiluminescent emission which was measured by a photomultiplier. The inter-assay and intra-assay coefficients of variation were $8.33 \%$ and $6.25 \%$, respectively. (2) $\mathrm{T}_{3}$ and $\mathrm{T}_{4}$ : A competition principle was used. Samples and $\mathrm{T}_{3} / \mathrm{T}_{4}$-specific antibodies labelled with a ruthenium complex; bound $\mathrm{T}_{3} / \mathrm{T}_{4}$ was released from the binding proteins in the sample by 8-Anilino-1-naphthalenesulfonic acid (ANS). After addition of streptavidin-coated microparticles and biotinylated $\mathrm{T}_{3} / \mathrm{T}_{4}$, the still-free binding sites of the labelled antibody became occupied, with formation of an antibody-hapten complex. The entire complex became bound to the solid phase via interaction of biotin and streptavidin. The reaction mixture was aspirated into the measuring cell where the microparticles were magnetically captured onto the surface of the electrode. Unbound substances were then removed with ProCell/ProCell M. Application of a voltage to the electrode then induced chemiluminescent emission which was measured by a photomultiplier. The inter-assay and intra-assay coefficients of variation were below $8.33 \%$ and $6.25 \%$ for $\mathrm{T}_{3}, 6.67 \%$ and $5.00 \%$ for $\mathrm{T}_{4}$, respectively. (3) TPOAb and TgAb: A competition principle was used. Samples were incubated with anti-TPO/Tg antibodies labelled with a ruthenium complex. After the addition of biotinylated TPO/Tg and strepdtavidin-coated microparticles, the anti-TPO/Tg antibodies in the sample competed with the ruthenium-labelled anti-TPO/Tg antibodies for the biotinylated TPO/Tg antigen. The entire complex became bound to the solid phase via the interaction of biotin and streptavidin. The reaction mixture was aspirated into the measuring cell where the microparticles were magnetically captured onto the surface of the electrode. Unbound substances were then removed with ProCell/ProCell M. The application of a voltage to the electrode then induced chemiluminescent emission, which was measured by a photomultiplier. The inter-assay and intra-assay coefficients of variation for both TPOAb and $\mathrm{TgAb}$ were $10.00 \%$ and $7.50 \%$, respectively.

Whole-blood $\mathrm{Pb}$ and $\mathrm{Cd}$ concentrations were tested using graphite furnace atomic absorption spectrometry (GFAAS, BH2200S, China). Before sample detection, a standard solution was tested in the machine, and then standard curves were set up with $r \geq 0.9950$. In the next step, quality control materials were tested, and the outcomes had to be within the range of accuracy ratings. After that, $40 \mu \mathrm{L}$ of whole blood was added to a reagent matched to the GFAAS machine. This reagent specialises in testing cadmium and lead in whole blood, which contained $\mathrm{NH}_{4} \mathrm{H}_{2} \mathrm{PO}_{4}+$ bovine + Triton X-100 + deionised water. After sufficient mixing, the solution can stand for half an hour. After that, $20 \mu \mathrm{L}$ samples were added to the inspection wells for testing. Two quality control personnel participated in the whole process control. Outliers were tested in duplicate. The limit of detection values for $\mathrm{BPb}$ and BCd were as follows: $1.17 \mu \mathrm{g} / \mathrm{L}$ and $0.02 \mu \mathrm{g} / \mathrm{L}$. None of the samples exhibited values below the detection limits of $\mathrm{BPb}$ and $\mathrm{BCd}$. The inter-assay and intra-assay coefficients of variation for $\mathrm{BPb}$ and $\mathrm{BCd}$ were all below $10.00 \%$.

Spot urine was obtained from each participant in the same morning. Urinary iodine and creatinine $(\mathrm{Cr})$ concentration measurements began on the day when samples arrived in the laboratory. Using the morning fasting spot urine samples, urine iodine concentration (UIC) was determined with an inductively coupled plasma-mass spectrometry (Agilent Technologies, Agilent $7700 \mathrm{X}, \mathrm{USA}$ ). After the sample solution was treated with tetramethylammonium hydroxide, it was sent from the carrier gas into the inductively coupled plasma torch through atomisation, and after evaporation, dissociation, atomisation, ionisation and other processes, most of it was converted into positively charged positive ions entering the mass spectrometer through the ion acquisition system. The mass spectrometer separated the sample according to the mass-to-charge ratio, which was detected by the detector. The ion count rate was proportional to the content of the analyte in the sample. The matrix effect was eliminated by the standard addition method to realise the sample quantitative analysis of iodine content. The inter-assay and intra-assay coefficients of variation for UIC were below $3.21 \%$ and $1.46 \%$, respectively.

\section{Thyroid ultrasonography}

All thyroid ultrasound examinations in Shanghai and Yunnan were performed by the same two registered physicians, who had professional certificates for ultrasonography awarded by the Ministry of Health of China, using B-mode US imaging. The internal echo of the thyroid was recorded. Prior to the study, strict ultrasound quality control was carried out, and the Kappa value between these two sonographers was 0.86 .

\section{Definition of variables}

Serum TPOAb positivity (TPOAb+) and TgAb positivity $(\mathrm{TgAb}+)$ were defined as TPOAb $>34.00 \mathrm{U} / \mathrm{mL}$ and $\mathrm{TgAb}>115.00 \mathrm{U} / \mathrm{mL}$ according to the reference range.

Thyroid autoimmune status was defined in two different ways: (1) ATA positive (ATA+): serum TPOAb + or TgAb+; and (2) ATA + and US+: serum TPOAb + or TgAb + together with characteristic ultrasonographic features (diffuse parenchymal hypoechogenicity and/or heterogeneous echogenic patterns of the thyroid gland). ${ }^{14} 15$

Hypothyroid status was defined as participants with higher TSH levels $(>4.20 \mathrm{mIU} / \mathrm{L})$ or with a history of thyroxine replacement therapy. 
Body mass index (BMI) was calculated as weight in kilograms divided by height in metres squared. UI/Cr $(\mu \mathrm{g} / \mathrm{g})=\mathrm{UIC}(\mu \mathrm{g} / \mathrm{L}) \times 88.4 \times 100 / \mathrm{UCr}(\mu \mathrm{mol} / \mathrm{L})$.

\section{Statistical analysis}

We performed survey analyses with IBM SPSS Statistics, V.24 (IBM Corporation). All analyses were two-sided. Value $\mathrm{p}<0.05$ was considered statistically significant. The Kolmogorov-Smirnov test and P-P plots were used to determine whether the data were normally distributed. Normally and non-normally distributed continuous variables were expressed as the mean $\pm \mathrm{SD}$ and the median (25\% quartile value, $75 \%$ quartile value), respectively. Categorical variables are presented as percentages.

The standardised prevalence of TPOAb+, TgAb+, ATA+, ATA + and US+ and hypothyroid status in Shanghai, Yunnan and four ethnic groups in Yunnan (Han, Yi, Miao and Hani) was analysed according to the age and sex distribution in the Sixth National Population Census of China, 2010 (National Bureau of Statistics, www. stats.gov.cn). The association of the prevalence of these thyroid disorders above with two locations (Shanghai and Yunnan) and four ethnic groups (Han, both in Shanghai and Yunnan and Yi, Miao, and Hani in Yunnan) was analysed by logistic regression. The regression models were adjusted for age, sex, location, ethnic group, BMI, smoking and drinking history (current and past). Han ethnicity was the reference.

The relationship of TPOAb+, TgAb+, ATA+, ATA + and US+, hypothyroid status with environmental parameters (UIC, urinary iodine-to-creatinine ratio (UI/Cr), $\mathrm{BPb}$ and BCd quartile concentration) was analysed by logistic regression models with each measure as the outcome. The regression models were adjusted for age, sex, ethnic group, BMI, smoking and drinking history (including current and past). The results were expressed as ORs and 95\% CIs. Levels of UIC and UI/Cr were divided into four groups: Level 1:<100.00, Level 2: 100.00-199.99, Level 3: 200.00-299.99 and Level 4: $\geq 300.00 \mu \mathrm{g} / \mathrm{L}$ ( or $\mu \mathrm{g} / \mathrm{g}$ ) according to the iodine nutrition epidemiological criteria of the WHO. ${ }^{16}$ For UIC and UI/Cr, the Level 2 (adequate iodine intake) was the reference. For $\mathrm{BPb}$ and $\mathrm{BCd}$, the first quartile $(\mathrm{Q} 1)$ was the reference.

The association of thyroid hormones with environmental parameters was analysed in the participants without a history of using thyroid medicine. Linear regression was used, and the results were expressed as B values with $95 \%$ CIs. TSH and $\mathrm{T}_{3}$ were ln-transformed before linear regression because of their skewed distribution. The regression models were adjusted for age, sex, ethnic group, BMI, smoking and drinking history (current and past). For UIC and UI/Cr the Level 2 (adequate iodine intake) was the reference. For BPb and BCd, the first quartile $(\mathrm{Q} 1)$ was the reference.

\section{Patient and public involvement}

Patients and the public were not involved in the development of research questions, design of the study, recruitment and conduct of the study or dissemination of the study results.

\section{RESULTS}

\section{Characteristics according to locations and ethnic groups}

A total of 2877 subjects (2105 in Shanghai and 772 in Yunnan), aged 18-75, were enrolled in the current study. All participants in Shanghai were Han. Four ethnic groups (Han and three ethnic minorities (Yi, Miao, and Hani)) in Yunnan, the region with the most ethnic minorities in China, were included in the current study. The characteristics of the participants in Shanghai, Yunnan and four ethnic groups in Yunnan are shown in table 1. The median (25\% quartile value, $75 \%$ quartile value) UIC in Shanghai and Yunnan was $199.90(142.65,291.60) \mu \mathrm{g} / \mathrm{L}$ and $213.20(150.00,291.30) \mu \mathrm{g} / \mathrm{L}$, respectively. The median (25\% quartile value, $75 \%$ quartile value) $\mathrm{BPb}$ was $35.00(28.00,43.00) \mu \mathrm{g} / \mathrm{L}$ in Shanghai and 49.00 (34.00, $68.00) \mu \mathrm{g} / \mathrm{L}$ in Yunnan. The median (25\% quartile value, $75 \%$ quartile value) BCd was $0.80(0.50,1.20) \mu \mathrm{g} / \mathrm{L}$ in Shanghai and $1.20(0.70,1.80) \mu \mathrm{g} / \mathrm{L}$ in Yunnan. Among the four ethnic groups in Yunnan, Han people had the highest median levels of UIC, BPb and BCd, while Hani people had the lowest median levels (table 1).

Standardised prevalence of thyroid status in the current study Figure 2 shows the standardised prevalence of thyroid status including the presence of TPOAb+, TgAb+, ATA+, ATA + and US+ and hypothyroid status in Shanghai (all were Han), Yunnan and four ethnic groups in Yunnan based on the age and sex distribution in The Sixth National Population Census of China, 2010. The standardised prevalence of thyroid disorders was much higher in Yunnan than in Shanghai (TPOAb+: $13.56 \%$ vs $8.27 \%$, $\mathrm{p}<0.001$; TgAb+: $9.28 \%$ vs $7.09 \%, \mathrm{p}=0.045$; ATA+: $16.96 \%$ vs $11.10 \%, \mathrm{p}<0.001$; ATA + and US+: $8.96 \%$ vs $6.64 \%$, $\mathrm{p}=0.036$; hypothyroid status: $13.53 \%$ vs $12.61 \%, \mathrm{p}=0.531$ ). In terms of the standardised prevalence of thyroid disorders in four ethnic groups in Yunnan, the Han population in Yunnan ranked first in TPOAb+, TgAb+, ATA+, $\mathrm{ATA}+$ and US+ and hypothyroid status.

\section{Association between the prevalence of thyroid status and demographic characteristics}

The association between the prevalence of thyroid status and demographic characteristics was analysed by using logistic regression models. The regression models were adjusted for age, sex, location, ethnic group, BMI, smoking and drinking history. Women were significantly associated with a higher prevalence of TPOAb+ (OR 2.219, $95 \%$ CI 1.453 to $3.387, \mathrm{p}<0.001)$, TgAb+ (OR 4.968, $95 \%$ CI 2.786 to $8.859, \mathrm{p}<0.001$ ), ATA+ (OR $3.128,95 \%$ CI 2.099 to $4.661, \mathrm{p}<0.001$ ), ATA + and US+ (OR 4.007, $95 \%$ CI 2.334 to $6.879, \mathrm{p}<0.001)$ and hypothyroid status (OR 2.197, 95\% CI 1.550 to 3.114, p<0.001). Age was significantly associated with a higher prevalence of hypothyroid status (OR 1.027, 95\% CI 1.017 to 1.037 , p<0.001). 


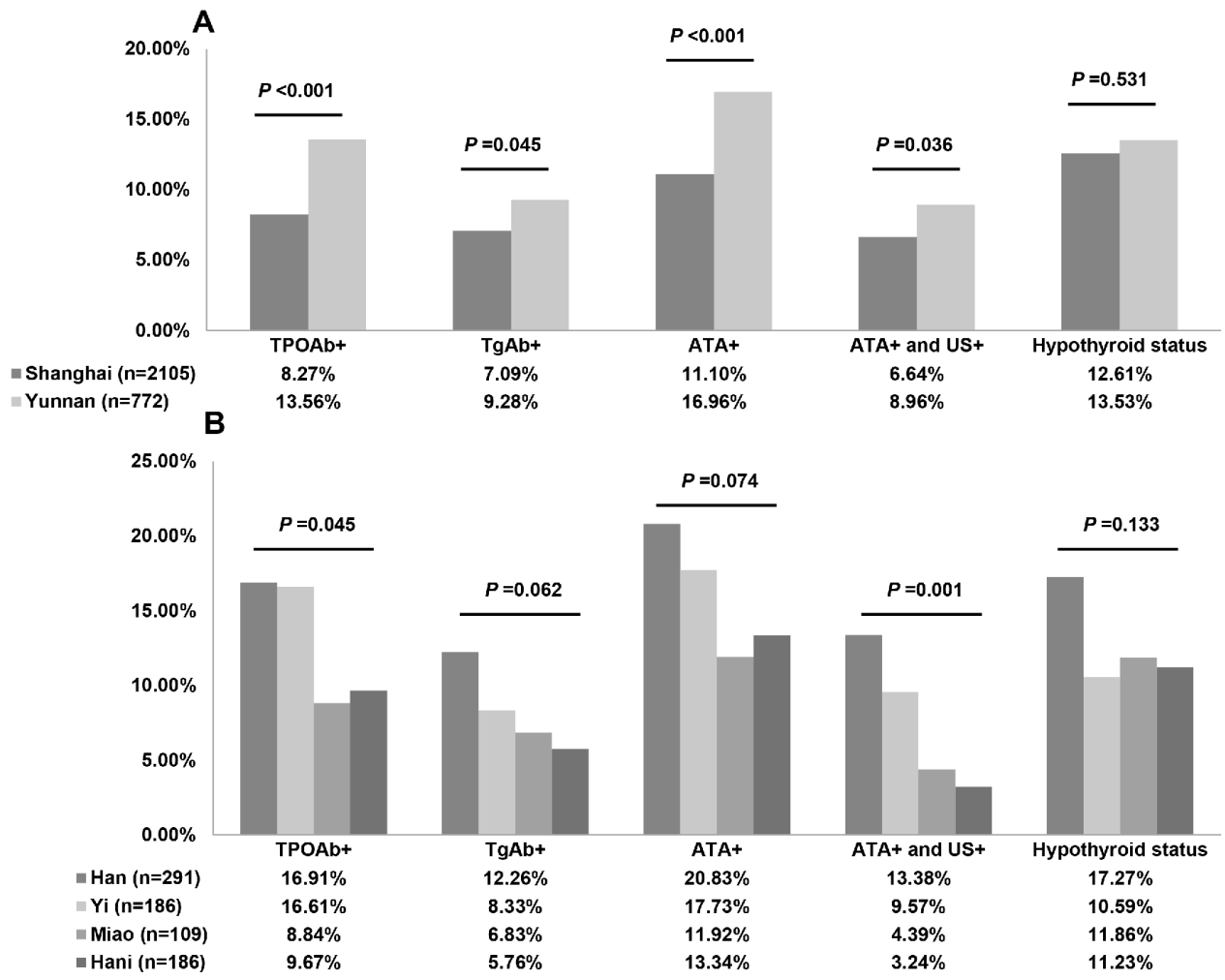

Figure 2 Standardised prevalence of thyroid disorders in the current study. (A) The standardised prevalence of thyroid disorders was significantly higher in Yunnan than in Shanghai. (B) The standardised prevalence of thyroid disorders in four ethnic groups in Yunnan, the Han population ranked first in TPOAb+, TgAb+, ATA+, ATA + and US+ and hypothyroid status. The standardised prevalence of thyroid disorders was based on the age and sex distribution in The Sixth National Population Census of China (2010). ATA+: serum TPOAb +or TgAb+; ATA+ and US+: serum TPOAb+ or TgAb+ together with characteristic ultrasonographic features; hypothyroid status: with higher thyroid stimulating hormone levels $(>4.20 \mathrm{mIU} / \mathrm{L})$, or with a history of thyroxine replacement therapy. ATA, antithyroid antibody; TgAb, thyroglobulin antibody; TPOAb, thyroid peroxidase antibody; US, ultrasound.

BMI, smoking history and drinking history showed no statistically significant association with thyroid autoimmunity and hypothyroid status (all $\mathrm{p}>0.05$ ) (table 2 ).

Table 2 also summarises the results of the association of TPOAb+, TgAb+, ATA+, ATA + and US+ and hypothyroid status with location and ethnic group. Participants in Yunnan had a 2.4-fold $(\mathrm{p}<0.001), 1.8$-fold $(\mathrm{p}=0.005), 2.2$ fold $(p<0.001), 2.2$-fold $(p<0.001)$ and 1.6-fold $(p=0.005)$ higher risk of developing TPOAb+, TgAb+, ATA+, ATA + and US+ and hypothyroid status than those in Shanghai. For ethnic groups, by using Han as a reference, a significant difference was seen in the prevalence of ATA + and US+ and hypothyroid status ( $\mathrm{p}=0.010$ and 0.037 ). Compared with the Han population, the Miao and Hani populations had lower risks of developing ATA + and US+, while the Yi and Hani populations had lower risks of developing hypothyroid status (all $\mathrm{p}<0.05)$.

\section{Association of environmental parameters with thyroid autoimmunity}

We further analysed the impact of environmental factors (mainly iodine, $\mathrm{Cd}$ and $\mathrm{Pb}$ ) on thyroid autoimmunity. We evaluated the adjusted ORs for TPOAb+, TgAb + and ATA+, ATA + and US+ according to the levels of UIC, UI/ Cr by using adequate iodine intake level (Level 2) as a reference, and the quartiles of $\mathrm{BPb}$ and BCd by using Q1 as a reference (table 3). For UI/Cr, compared with Level 2, Level 4 (excessive iodine intake) had a 1.5-fold risk for ATA + and US+ (OR 1.455, 95\% CI 1.015 to 2.087). For $\mathrm{BPb}$, compared with the first quartiles, the highest quartiles had highest risks of TPOAb+, ATA+, ATA + and US+ (TPOAb+: OR 1.637, 95\% CI 1.153 to 2.322; ATA+: OR $1.435,95 \%$ CI 1.046 to 1.968 ; ATA + and US+: OR 1.641, $95 \%$ CI 1.112 to 2.422$)$. For BCd, the fourth quartile was also significantly positively associated with the prevalence of ATA+ (OR 1.427, 95\% CI 1.023 to 1.991 ).

\section{Association of environmental parameters with thyroid function}

Hypothyroid status was defined as participants with higher TSH levels $(>4.20 \mathrm{mIU} / \mathrm{L})$ or with a history of thyroxine replacement therapy. According to this definition, we found that Level 3 (above requirements iodine intake) and Level 4 (excessive iodine intake) of UI/Cr was positively associated with the prevalence of hypothyroid status (Level 3: OR 1.509, 95\% CI 1.165 to 1.954; Level 4: OR 1.338, 95\% CI 1.010 to 1.772) (table 3). For $\mathrm{BPb}$, the fourth quartiles of $\mathrm{BPb}$ were positively associated with higher risk of hypothyroid status (OR 1.467, 95\% CI 1.085 to 1.984 , table 3 ) and TSH levels (B 0.092, 


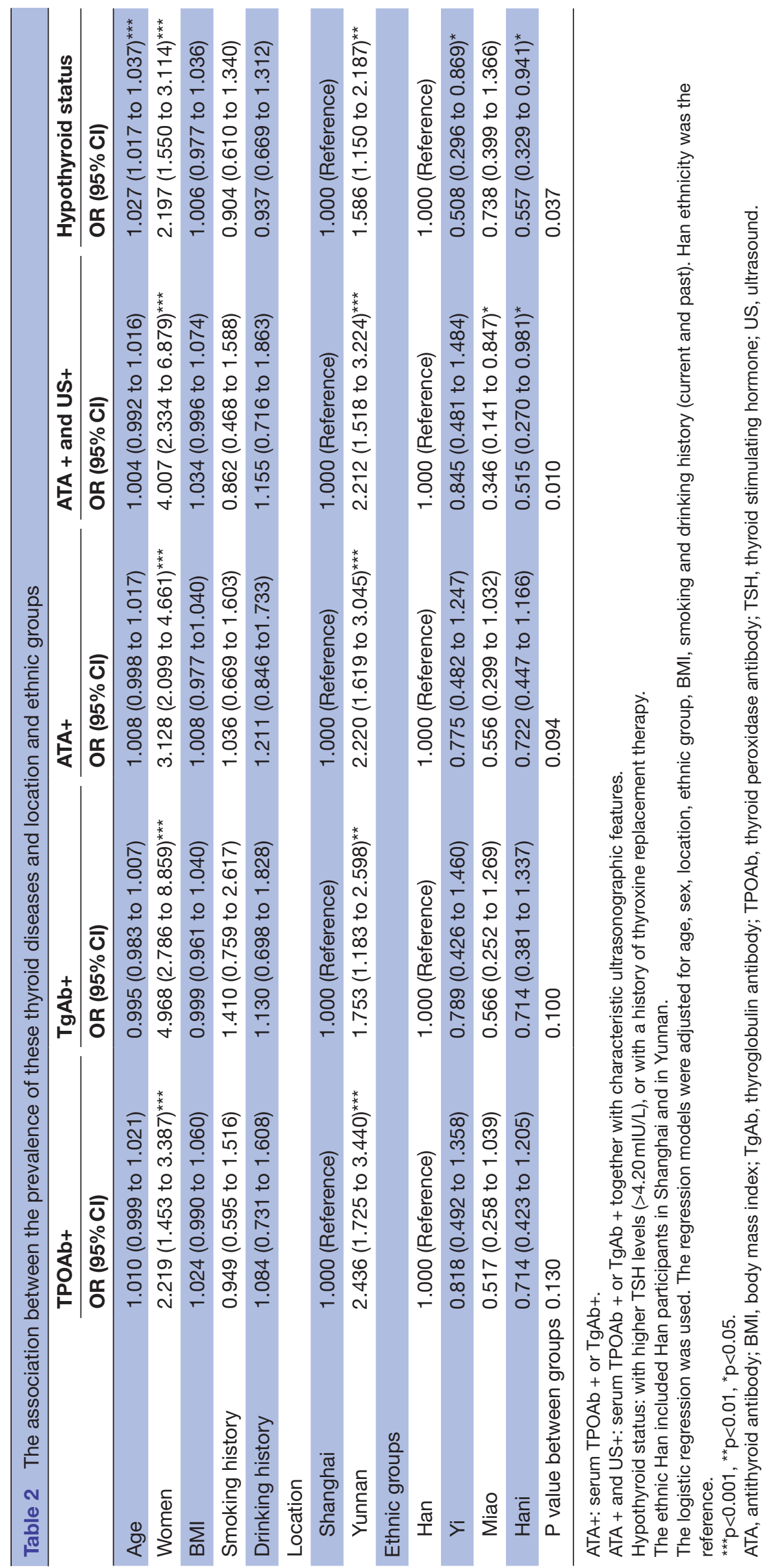




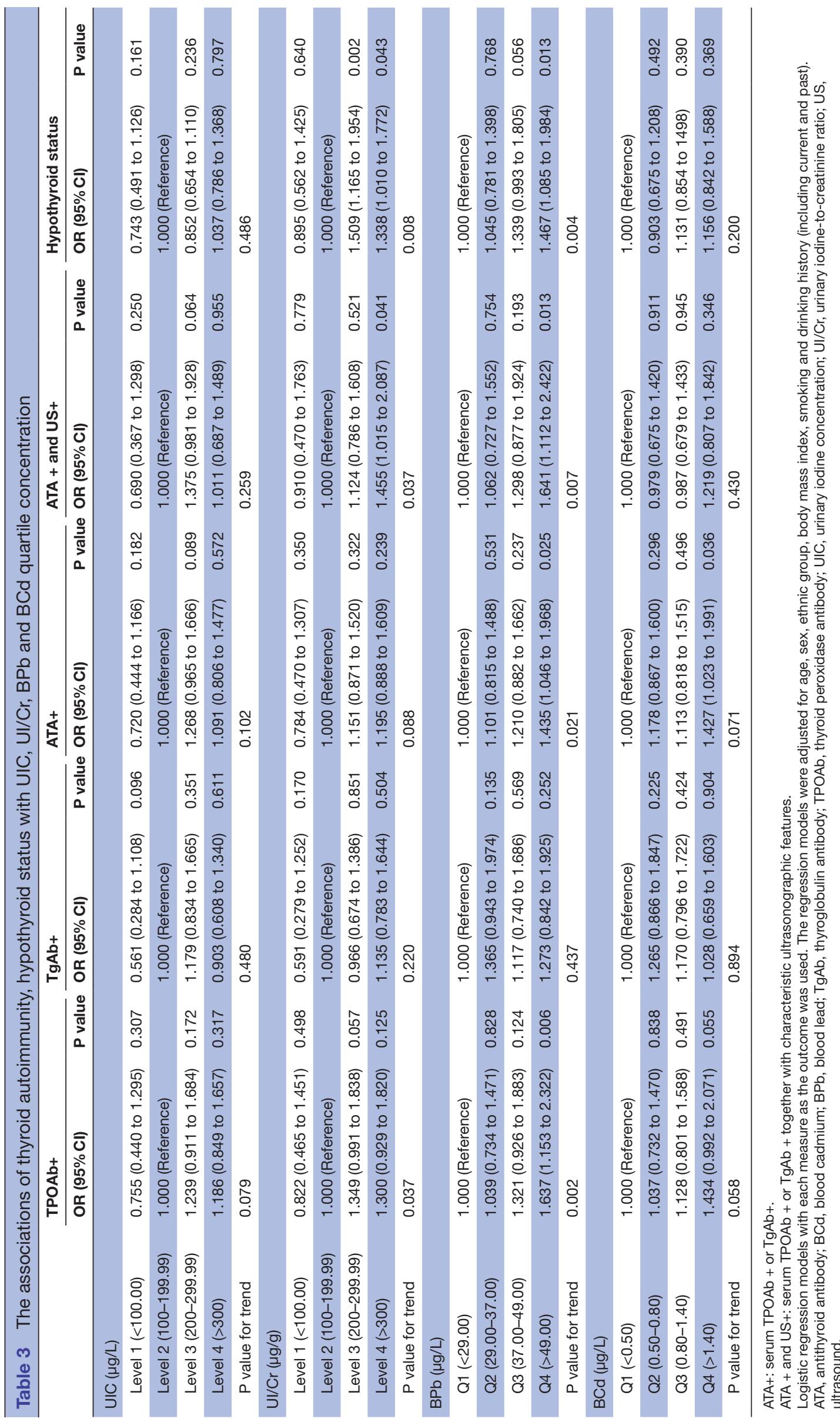


Table 4 The associations of thyroid hormones with $\mathrm{UIC}, \mathrm{UI} / \mathrm{Cr}, \mathrm{BPb}$ and $\mathrm{BC}$ quartile concentration

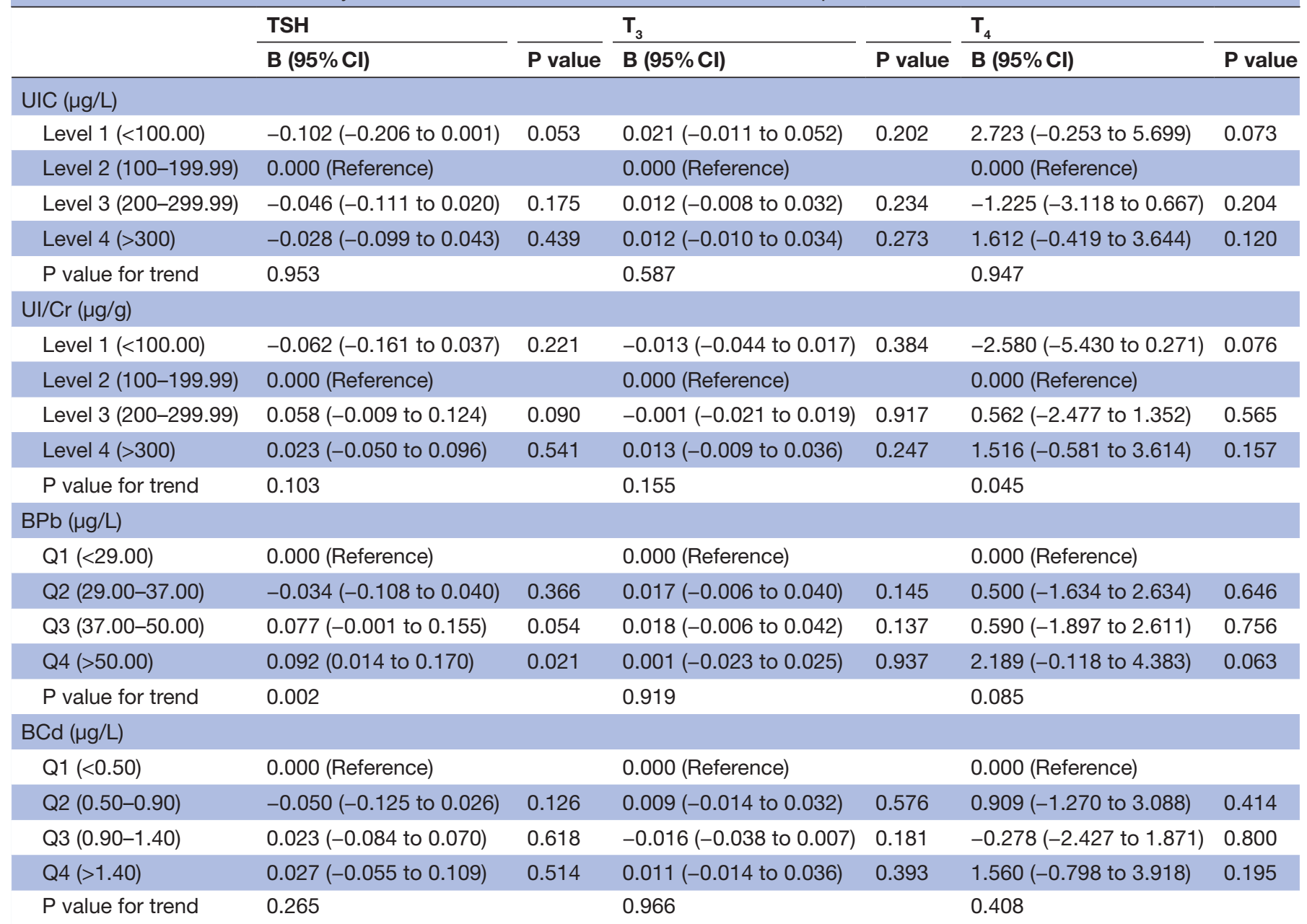

Linear regression was used. TSH and $\mathrm{T}_{3}$ were In-transformed before linear regression because of their skewed distribution. The regression models were adjusted for age, sex, ethnic group, body mass index, smoking and drinking history (current and past). BCd, blood cadmium; BPb, blood lead; T3, triiodothyronine; T4, total thyroxin; TSH, thyroid stimulating hormone; UIC, urinary iodine concentration; $\mathrm{UI} / \mathrm{Cr}$, urinary iodine-to-creatinine ratio.

95\% CI 0.014 to 0.170 , table 4$)$. There was no significant difference between BCd quartiles and the risk of thyroid dysfunction (tables 3 and 4).

\section{DISCUSSION}

In the current study, we found differences in the prevalence of thyroid autoimmune positivity, and hypothyroid status between two different regions (Shanghai and Yunnan) and among four different ethnic groups (Han, $\mathrm{Yi}, \mathrm{Miao}$ and Hani) in China. Higher levels of $\mathrm{UI} / \mathrm{Cr}, \mathrm{BPb}$ and BCd may be risk factors for thyroid autoimmunity and hypothyroid status.

Thyroid antibody positivity and AITDs have high prevalence in the general population. Several studies have reported that different races/ethnicities and socioeconomic statuses were associated with the prevalence of thyroid disorders. Using the data from the Third National Health and Nutrition Examination Survey (NHANES III) in the USA, Hollowell JG et alreported that TSH levels and the prevalence of ATAs were greater in whites and Mexican Americans than in blacks. ${ }^{17}$ In the Brazilian Longitudinal Study of Adult Health (ELSA-Brasil), the prevalence of hypothyroidism was greater in white individuals than in black individuals or Brazilians and among those with high net family incomes. ${ }^{18}$ In terms of ethnic differences, we found that in Yunnan Honghe Prefecture, Han people had the greatest standardised prevalence of thyroid autoimmune positivity and hypothyroid status. Compared with the Han people living in Yunnan, the Yi and Miao people are still in a relatively poor state (especially in remote rural areas). Their eating habits are mostly vegetarian, and the amount of crude fibre and grains they consume is significantly higher than that the amount that Han people in Yunnan consume. It was reported that some vegetables, such as cruciferous vegetables, pearl millet, soy products and cassava, were attributed to thyroid dysfunction. ${ }^{19}$ The Han ethnicity shows high genetic homogeneity across China, and significant genetic differences exist between 
Han groups and some minority groups. ${ }^{20}$ However, it is worth noting that in the same Han population living in Shanghai (one of the most economically developed cities in China) or in Yunnan in different locations, the standardised prevalence of thyroid disorders presented was completely different. The prevalence of thyroid autoimmune positivity and hypothyroid status was much higher in Yunnan Han people than in Shanghai Han people. Thus, in addition to potential genetic differences, differences in eating habits and regional economic differences, potential environmental risk factors may also contribute to the different prevalence of thyroid disorders.

Iodine is a trace element essential for the human body, especially for thyroid hormone production. It is primarily obtained from the diet and excreted in urine. The measurement of UIC is recommended by the WHO in evaluating iodine nutrition among populations, ${ }^{16}$ and according to the iodine nutrition epidemiological criteria of the WHO, a population's median UIC of $<100,100-199$, 200-299 and $\geq 300 \mu \mathrm{g} / \mathrm{L}$ are each representative of insufficient, adequate, above requirements and excessive iodine intake. Some studies also used UI/Cr to describe iodine status. ${ }^{21}{ }^{22}$ It was suggested that UI/Cr from spot urine can serve as a feasible and reliable alternative for evaluating iodine excretion when a 24-hour urine sample is unavailable. ${ }^{16}$ Thus, in the current study, we used these two methods to assess the iodine nutritional status of the population. In our study, the median (25\% quartile value, $75 \%$ quartile value) UICs of the populations in Shanghai and Yunnan were 199.90 (142.65, 291.60) $\mu \mathrm{g} / \mathrm{L}$ (adequate) and $213.20(150.00,291.30) \mu \mathrm{g} / \mathrm{L}$ (above requirement), respectively. Although Shanghai is a coastal city and Yunnan is an inland area, the UIC levels of the Shanghai population should have been higher than those of the Yunnan population. However, a survey of 12 communities in downtown Shanghai found that approximately $46.4 \%$ of Shanghai residents currently use noniodised salt, ${ }^{23}$ and $\mathrm{UIC}<100 \mu \mathrm{g} / \mathrm{L}$ was present in $46.8 \%$ of the population. That is why the UIC level of the Shanghai population in the current study was slightly lower than that of the Yunnan population.

In the current study, we evaluated the association of UIC and UI/Cr with thyroid autoimmunity and hypothyroid status by using adequate iodine intake level (Level 2 ) as a reference. We found excessive iodine intake had a 1.5-fold risk for ATA + and US+, and above requirements iodine intake and excessive iodine intake was positively associated with hypothyroid status. Many studies have focused on the problems of excessive iodine intake. Excessive iodine consumption has been widely described as a risk factor for AITD. ${ }^{24}{ }^{25}$ In a 5 -year prospective Chinese study, ${ }^{26}{ }^{27}$ the cumulative incidence of ATA was significantly higher in the cohort with excessive iodine intake. Excessive iodine intake is also related to a higher prevalence of hypothyroidism or an increased level of $\mathrm{TSH},{ }^{28-31}$ and the hypothyroid status following chronic excess iodine exposure may be due to the presence of ATA positivity. ${ }^{26} 2732$
Lead $(\mathrm{Pb})$ and cadmium $(\mathrm{Cd})$ are inevitable environmental pollutants in daily life. They come from a variety of sources, such as leaded gasoline, mining wastes, smelting, electroplating, petroleum and lead paint, as well as the intensive use of consumer products, such as lead-acid batteries, make-up, pigments, plastic cooking tools and cigarettes. ${ }^{33-35}$ The biological half-life of Cd and $\mathrm{Pb}$ can be over 30 years. Thus, exposure to $\mathrm{Cd}$ and $\mathrm{Pb}$, even at environmentally low levels over time, is associated with a plethora of toxic effects on multiple systems and organs of the human body, ${ }^{36}$ including endocrinedisrupting activities. ${ }^{37} \mathrm{BPb}$ and $\mathrm{BCd}$ levels are widely used as biomarkers and are included in human biomonitoring related to $\mathrm{Pb}$ and $\mathrm{Cd}$ exposure in many countries. In the NHANES (2007-2010) of the USA, ${ }^{38} \mathrm{BPb}$ and BCd were $18.20 \mu \mathrm{g} / \mathrm{L}$ and $0.55 \mu \mathrm{g} / \mathrm{L}$, respectively, and in Asia, $\mathrm{BPb}$ and BCd were $14.60 \mu \mathrm{g} / \mathrm{L}$ and $0.72 \mu \mathrm{g} / \mathrm{L}$, respectively, in the Korean National Health and Nutrition Examination Survey (KNHANES, 2017). ${ }^{39}$ In the current study, the median levels of $\mathrm{BPb}$ and $\mathrm{BCd}$ were $35.00 \mu \mathrm{g} / \mathrm{L}$ and $0.80 \mu \mathrm{g} / \mathrm{L}$ in Shanghai, $49.00 \mu \mathrm{g} / \mathrm{L}$ and $1.20 \mu \mathrm{g} / \mathrm{L}$ in Yunnan, respectively, and they were slightly higher than those data reported in the other countries above.

Lead $(\mathrm{Pb})$ and cadmium $(\mathrm{Cd})$ are also recognised as endocrine-disrupting chemicals. ${ }^{37}{ }^{40}$ Some reports suggested that the frequency of TPOAb positivity in never smokers was significantly higher than that in ever smokers ${ }^{41}$ and that stopping smoking decreases the risk of Graves' disease but increases the risk of Hashimoto disease. ${ }^{42}$ However, in the current study, smoking history showed no significant association with thyroid autoimmunity and hypothyroid status, considering that smokers had higher levels of $\mathrm{BPb}$ and BCd than non-smokers, ${ }^{43} 44$ and all analyses in the current study were performed with adjustment for smoking. The relationship between $\mathrm{Cd}$ and $\mathrm{Pb}$ exposure and thyroid disorders remains controversial. Abdelouahab et $a t^{45}$ studied a group of 124 men (median BPb: $31.0 \mu \mathrm{g} / \mathrm{L}$ ) and 87 women (median BPb: $17.4 \mu \mathrm{g} / \mathrm{L}$ ) who lived in lakeside communities. They found an inverse relationship between $\mathrm{TSH}$ and $\mathrm{BPb}$ in women but not in men. Meeker $e t a t^{46}$ found that TSH decreased as the whole $\mathrm{BPb}$ concentration increased in 219 men participating in a study of environmental influences on male reproductive health (median $\mathrm{BPb}: 15.0 \mu \mathrm{g} / \mathrm{L}$ ). The results from the analysis of the Third NHANES III in the USA showed that the TSH level was not significantly related to the $\mathrm{BPb}$ concentration (mean BPb: $35.5 \mu \mathrm{g} / \mathrm{L}$ ) ${ }^{47}$ Similar results have been obtained in two other studies ${ }^{38} 48$ (mean $\mathrm{BPb}: 15.5 \mu \mathrm{g} / \mathrm{L}$ and $18.2 \mu \mathrm{g} / \mathrm{L}$, respectively). However, in our study, increasing quartiles of $\mathrm{BPb}$ were positively associated with increasing risks of thyroid autoimmunity and hypothyroid status, including increased TSH levels. Regarding Cd, Buha et al reported that elevated BCd levels were associated with decreased TSH levels. ${ }^{36}$ One study on workers occupationally exposed to Cd (median BCd: $0.71 \mu \mathrm{g} / \mathrm{L}$ ) confirmed that higher BCd concentrations amplify the risk of elevated TSH levels. ${ }^{49}$ Chen $e t a \tilde{l}^{0}$ analysed the results of NHANES (2007-2008) data and 
suggested that increased Cd levels (mean BCd: $0.38 \mu \mathrm{g} / \mathrm{L}$ ) were associated with increased $\mathrm{Tg}$ levels, but levels of TSH were not consistently associated with Cd exposure. In another study using NHANES (2007-2008) data, ${ }^{51}$ but using different statistical methods in a relatively small population (median BCd: $0.30 \mu \mathrm{g} / \mathrm{L}$ ), elevated BCd levels were associated with decreased TSH levels. Recent literature tackling the topic of biphasic, or non-monotonic, responses to $\mathrm{Cd}$ exposure was characterised by an inverted U-shaped curve. ${ }^{52}$ In our study, a positive relationship was found only between the highest quartile of BCd and thyroid autoimmunity, and no significant association was found between Cd exposure and thyroid function. Collectively, human studies have yielded conflicting data, leading to increased confusion about the relationships between $\mathrm{BPb}$ and $\mathrm{BCd}$ and thyroid disorders. These findings may be due to variations in participant selection and the use of different statistical methods.

Our study has several strengths. First, there were few reports on the levels of heavy metals and thyroid autoimmunity and hypothyroid status in multiple ethnic groups and regions. Second, all anthropometric measurements, questionnaires and ultrasonography were completed by the same trained research group with strong quality control, which minimising the inter-individual errors.

However, some limitations must be taken into account. First, because of the cross-sectional nature of the current study, association does not indicate causation. Second, $\mathrm{BCd}$ and $\mathrm{BPb}$ concentrations were based on a single blood sample, and they may represent short-term exposure. Third, due to the relatively conservative cultural background, inconvenient transportation (mountain areas) and the impact of COVID-19, the sample size of four different ethnics in Yunnan was relatively small. This pure exploratory research could be regarded as a pilot experiment. In the future, we will deepen our cooperation in Yunnan and expand our sample size to explore more results.

\section{CONCLUSION}

Higher levels of UI/Cr, $\mathrm{BPb}$ and BCd may be associated with thyroid autoimmunity and hypothyroid status. Understanding the epidemiology of thyroid autoimmunity, including its geographical differences and environmental influences, may provide clues to find its aetiology.

Acknowledgements The authors thank all team members from Institute and Department of Endocrinology and Metabolism, Shanghai Ninth People's Hospital, Shanghai Jiaotong University School of Medicine (Shanghai, China); Department of Endocrinology, the Fifth affiliated Hospital of Kunming Medical University, Yunnan Honghe Prefecture Central Hospital (Ge Jiu People's Hospital) (Yunnan, China).

Contributors $Y L$ designed the study and responsible for the overall content as guarantor; YChen, QX, NW, WZ, CZ, YW, HW, JC and KZ participated in acquisition of data; $Y L$ and $Y C a i$ evaluated the literature. $Y C h e n$ and $Q X$ undertook the statistical analysis, wrote the first draft of the manuscript and revised manuscript. All authors read and approved the final manuscript for publication.

Funding The current study was supported by National Natural Science Foundation of China (81800694, 91857117); Yunnan Province Lu Yingli Expert Workstation; Yunnan Provincial Key Subject Project; Science and Technology Commission of
Shanghai Municipality (19140902400, 18410722300); the Major Science and Technology Innovation Programme of Shanghai Municipal Education Commission (2019-01-07-00-01-E00059); Commission of Health and Family Planning of Pudong District (PWZxq2017-17); Shanghai JiaoTong University School of Medicine (19XJ11007). The funders played no role in the design or conduct of the study, collection, management, analysis or interpretation of data or in the preparation, review or approval of the article.

Competing interests None declared.

Patient and public involvement Patients and/or the public were not involved in the design, or conduct, or reporting, or dissemination plans of this research.

Patient consent for publication Not applicable.

Ethics approval These two study protocols were both approved by the Ethics Committee of Shanghai Ninth People's Hospital, Shanghai JiaoTong University School of Medicine (SH9H-2019-T187-2, SH9H-2019-T107-1). Participants gave informed consent to participate in the study before taking part.

Provenance and peer review Not commissioned; externally peer reviewed.

Data availability statement All data relevant to the study are included in the article or uploaded as supplementary information.

Open access This is an open access article distributed in accordance with the Creative Commons Attribution Non Commercial (CC BY-NC 4.0) license, which permits others to distribute, remix, adapt, build upon this work non-commercially, and license their derivative works on different terms, provided the original work is properly cited, appropriate credit is given, any changes made indicated, and the use is non-commercial. See: http://creativecommons.org/licenses/by-nc/4.0/.

ORCID iD

Yingli Lu http://orcid.org/0000-0002-5117-1614

\section{REFERENCES}

1 Orgiazzi J. Thyroid autoimmunity. Presse Med 2012;41:e611-25.

2 Jacobson DL, Gange SJ, Rose NR, et al. Epidemiology and estimated population burden of selected autoimmune diseases in the United States. Clin Immunol Immunopathol 1997;84:223-43.

3 Pearce SHS, Leech NJ. Toward precise forecasting of autoimmune endocrinopathy. J Clin Endocrinol Metab 2004;89:544-7.

4 Brčić L, Barić A, Gračan S, et al. Association of established thyroid peroxidase autoantibody (TPOAb) genetic variants with Hashimoto's thyroiditis. Autoimmunity 2016;49:480-5.

5 Effraimidis G, Badenhoop K, Tijssen JGP, et al. Vitamin D deficiency is not associated with early stages of thyroid autoimmunity. Eur $J$ Endocrinol 2012;167:43-8.

6 Cogni G, Chiovato L. An overview of the pathogenesis of thyroid autoimmunity. Hormones 2013;12:19-29.

7 Massoudi MS, Meilahn EN, Orchard TJ, et al. Prevalence of thyroid antibodies among healthy middle-aged women. findings from the thyroid study in healthy women. Ann Epidemiol 1995;5:229-33.

8 Huber G, Staub J-J, Meier C, et al. Prospective study of the spontaneous course of subclinical hypothyroidism: prognostic value of thyrotropin, thyroid reserve, and thyroid antibodies. J Clin Endocrinol Metab 2002;87:3221-6.

9 Chen Y, Zhu C, Chen Y, et al. Are thyroid autoimmune diseases associated with cardiometabolic risks in a population with normal thyroid-stimulating hormone? Mediators Inflamm 2018;2018:1-10.

10 Chen Y, Wang N, Chen Y, et al. The association of non-alcoholic fatty liver disease with thyroid peroxidase and thyroglobulin antibody: a new insight from SPECT-China study. Autoimmunity 2018;51:238-44.

11 Hansen PS, Brix TH, lachine I, et al. The relative importance of genetic and environmental effects for the early stages of thyroid autoimmunity: a study of healthy Danish twins. Eur J Endocrinol 2006;154:29-38.

12 Brent GA. Environmental exposures and autoimmune thyroid disease. Thyroid 2010;20:755-61.

13 Antonelli A, Ferrari SM, Corrado A, et al. Autoimmune thyroid disorders. Autoimmun Rev 2015;14:174-80.

14 Chen Y, Chen Y, Xia F, et al. A higher ratio of estradiol to testosterone is associated with autoimmune thyroid disease in males. Thyroid 2017;27:960-6.

15 Muscogiuri G, Mari D, Prolo S, et al. 25 hydroxyvitamin D deficiency and its relationship to autoimmune thyroid disease in the elderly. Int $J$ Environ Res Public Health 2016;13:850.

16 Chen W, Li X, Guo X, et al. Urinary iodine excretion (UIE) estimated by iodine/creatinine ratio from spot urine in Chinese school-age children. Clin Endocrinol 2017;86:628-33. 
17 Hollowell JG, Staehling NW, Flanders WD, et al. Serum TSH, T(4), and thyroid antibodies in the United States population (1988 to 1994): National Health and Nutrition Examination Survey (NHANES III). J Clin Endocrinol Metab 2002;87:489-99.

18 Olmos RD, Figueiredo RCde, Aquino EM, et al. Gender, race and socioeconomic influence on diagnosis and treatment of thyroid disorders in the Brazilian longitudinal study of adult health (ELSABrasil). Braz J Med Biol Res 2015;48:751-8.

19 Babiker A, Alawi A, Al Atawi M, et al. The role of micronutrients in thyroid dysfunction. Sudan J Paediatr 2020;20:13-19.

20 Wang M, Wang Z, He G, et al. Genetic characteristics and phylogenetic analysis of three Chinese ethnic groups using the Huaxia platinum system. Sci Rep 2018;8:2429.

$21 \mathrm{Kim}$ HK, Lee SY, Lee JI, et al. Daily urine iodine excretion while consuming a low-iodine diet in preparation for radioactive iodine therapy in a high iodine intake area. Clin Endocrinol 2011;75:851-6.

22 Fuse Y, Ohashi T, Yamaguchi S, et al. lodine status of pregnant and postpartum Japanese women: effect of iodine intake on maternal and neonatal thyroid function in an iodine-sufficient area. J Clin Endocrinol Metab 2011;96:3846-54.

23 Chen $\mathrm{C}, \mathrm{Xu} \mathrm{H}$, Chen Y, et al. lodized salt intake and its association with urinary iodine, thyroid peroxidase antibodies, and thyroglobulin antibodies among urban Chinese. Thyroid 2017;27:1566-73.

24 Laurberg P, Cerqueira C, Ovesen L, et al. lodine intake as a determinant of thyroid disorders in populations. Best Pract Res Clin Endocrinol Metab 2010;24:13-27.

25 Ferrari SM, Fallahi P, Antonelli A, et al. Environmental issues in thyroid diseases. Front Endocrinol 2017;8:50.

26 Li Y, Teng D, Shan Z, et al. Antithyroperoxidase and antithyroglobulin antibodies in a five-year follow-up survey of populations with different iodine intakes. J Clin Endocrinol Metab 2008;93:1751-7.

27 Teng W, Shan Z, Teng X, et al. Effect of iodine intake on thyroid diseases in China. N Engl J Med 2006;354:2783-93.

$28 \mathrm{Kim} \mathrm{HI}$, Oh H-K, Park SY, et al. Urinary iodine concentration and thyroid hormones: Korea National health and nutrition examination survey 2013-2015. Eur J Nutr 2019;58:233-40.

29 Weng W, Dong M, Zhang J, et al. A PRISMA-compliant systematic review and meta-analysis of the relationship between thyroid disease and different levels of iodine intake in mainland China. Medicine 2017;96:e7279

30 Katagiri R, Yuan X, Kobayashi S, et al. Effect of excess iodine intake on thyroid diseases in different populations: a systematic review and meta-analyses including observational studies. PLOS One 2017; 12:e0173722.

31 Leung AM, Braverman LE. Consequences of excess iodine. Nat Rev Endocrinol 2014;10:136-42.

32 Wang $\mathrm{K}$, Zhang J, Li F, et al. Urinary iodine in early pregnancy is associated with subclinical hypothyroidism in Tianjin, China: an observational study. BMC Endocr Disord 2017:17:10.

33 Burman KD, Wartofsky L. Thyroid nodules. N Engl J Med 2016;374:1294-5.

34 Kahn LG, Liu X, Rajovic B, et al. Blood lead concentration and thyroid function during pregnancy: results from the Yugoslavia prospective study of environmental lead exposure. Environ Health Perspect 2014;122:1134-40.

35 Järup L. Hazards of heavy metal contamination. Br Med Bull 2003;68:167-82.
36 Buha A, Matovic V, Antonijevic B, et al. Overview of cadmium thyroid disrupting effects and mechanisms. Int J Mol Sci 2018:19:1501.

37 lavicoli I, Fontana L, Bergamaschi A. The effects of metals as endocrine disruptors. J Toxicol Environ Health B Crit Rev 2009;12:206-23.

38 Luo J, Hendryx M. Relationship between blood cadmium, lead, and serum thyroid measures in US adults - the National Health and Nutrition Examination Survey (NHANES) 2007-2010. Int J Environ Health Res 2014;24:125-36.

39 Ahn J, Kim N-S, Lee B-K, et al. Changes of atmospheric and blood concentrations of lead and cadmium in the general population of South Korea from 2008 to 2017. Int J Environ Res Public Health 2019:16:2096.

40 Chung SM, Moon JS, Yoon JS, et al. Sex-Specific effects of blood cadmium on thyroid hormones and thyroid function status: Korean nationwide cross-sectional study. J Trace Elem Med Biol 2019;53:55-61.

41 Mehran L, Amouzgar A, Delshad H, et al. The association of cigarette smoking with serum TSH concentration and thyroperoxidase antibody. Exp Clin Endocrinol Diabetes 2012;120:80-3.

42 Wiersinga WM. Clinical relevance of environmental factors in the pathogenesis of autoimmune thyroid disease. Endocrinol Metab 2016;31:213-22.

43 Snoj Tratnik J, Falnoga I, Mazej D, et al. Results of the first national human biomonitoring in Slovenia: trace elements in men and lactating women, predictors of exposure and reference values. Int $J$ Hyg Environ Health 2019;222:563-82.

44 Haines DA, Saravanabhavan G, Werry K, et al. An overview of human biomonitoring of environmental chemicals in the Canadian health measures survey: 2007-2019. Int J Hyg Environ Health 2017;220:13-28.

45 Abdelouahab N, Mergler D, Takser L, et al. Gender differences in the effects of organochlorines, mercury, and lead on thyroid hormone levels in lakeside communities of Quebec (Canada). Environ Res 2008;107:380-92.

46 Meeker JD, Rossano MG, Protas B, et al. Multiple metals predict prolactin and thyrotropin (TSH) levels in men. Environ Res 2009;109:869-73.

47 Krieg EF. The relationships between blood lead levels and serum thyroid stimulating hormone and total thyroxine in the third National health and nutrition examination survey. J Trace Elem Med Biol 2019;51:130-7.

48 Mendy A, Gasana J, Vieira ER. Low blood lead concentrations and thyroid function of American adults. Int $J$ Environ Health Res 2013:23:461-73.

49 Jurdziak M, Gać P, Poręba M, et al. Concentration of thyrotropic hormone in persons occupationally exposed to lead, cadmium and arsenic. Biol Trace Elem Res 2018:182:196-203.

50 Chen A, Kim SS, Chung E, et al. Thyroid hormones in relation to lead, mercury, and cadmium exposure in the National health and nutrition examination survey, 2007-2008. Environ Health Perspect 2013;121:181-6.

51 Yorita Christensen KL. Metals in blood and urine, and thyroid function among adults in the United States 2007-2008. Int J Hyg Environ Health 2013;216:624-32.

52 Zhang Y, Shen G, Yu Y, et al. The hormetic effect of cadmium on the activity of antioxidant enzymes in the earthworm Eisenia fetida. Environ Pollut 2009;157:3064-8. 